\title{
Sustaining the commons
}

\author{
No amount of engineering, laws or regulations will be sufficient to sustain the commons without a deeper \\ understanding of how, when and under what conditions humans cooperate.
}

T he Anthropocene has a bad reputation. Its geological status may be disputed, but the presumptive epoch has established itself in collective consciousness as synonymous with humanity wreaking havoc on the planet and its atmosphere. From biodiversity loss - at such rates as to support claims that we have entered the sixth mass extinction (Sci. Adv. 1, e1400253; 2015) - to land erosion and groundwater depletion, our legacy on this planet has been catastrophic, but it cannot continue to be so. We must act with urgency, but how do we effectively sustain the commons?

In some areas, technology has offered solutions. In several others, laws and regulations are put in place to effectively manage common resources. However, none of these is sufficient to avert the 'tragedy of the commons. Human behaviour is the crucial element: without cooperation and compliance, the commons will continue to be imperilled.

Ecologist Garrett Hardin argued that if people are left to their own devices, the tragedy of the commons is inevitable: people are driven by self-interest and, in maximizing their own marginal utility by over-exploiting a common resource, spoil it for the collective (Science 162, 1243-1248; 1968). Elinor Ostrom's view was far less bleak: in work that won her the only Nobel Prize in Economics to be awarded to a woman, she showed that on a local scale it is possible to manage a common resource in an economically and ecologically sustainable manner without privatization or regulation from central government.

Two papers in this issue of Nature Human Behaviour show under which conditions the tragedy of the commons will prevail, and those under which it will be averted.

Using economic games, Gächter et al. show that in repeated interactions among

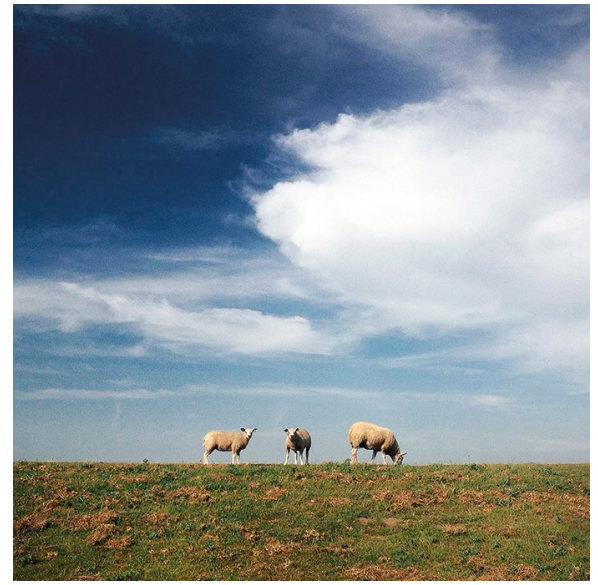

Credit: Room the Agency/Alamy Stock Photo

strangers, without any incentive to cooperate, the tragedy of the commons is almost absolute: by the end of the game, there is virtually no public good to speak of. Counterintuitively, their games and modelling show that it is much harder to maintain a public good than create a new one. People seem inclined to cooperate more when establishing a commons than while maintaining it. But when trying to maintain a public good, people are much more likely to punish those who do not cooperate.

The research presented by Castilla-Rho et al. is partly inspired by Ostrom's work and provides crucial insight into how sustainable groundwater management can be achieved in different cultural settings. The authors modelled farmers' use of groundwater for crop irrigation in three regions where groundwater extraction control is needed: the Murray-Darling Basin in Australia, the Central Valley in California, USA, and the Punjab on the India/Pakistan border. Their model incorporated data on social attitudes to cooperation and rule conformity in the different regions. The results show that strong punitive measures are likely to be effective in tightly knit, cooperative cultures (such as in the Punjab), but much less so in more individualistic cultures (in the USA and Australia). In fact, increased regulation and monitoring were found to have very limited impacts in individualistic societies in the model. Instead, across cultures, the most effective measure was increasing the number of 'good example' rule-followers in a population (although, between regions, there was strong variation in the number of rule-followers that was required to tip the scale towards a positive social norm).

These two papers offer important evidence when considering policies for commons management: clearly, one size does not fit all, and successful policies will need to be highly context dependent. Well-intended or not, the Western world is accustomed to exporting or prescribing its own 'tried and tested' policies in countries whose cultures and circumstances are wildly different. The research by Castilla-Rho et al. takes into account culturally variable social norms, demonstrating that this approach is likely to be futile. Maintaining a common resource is much harder than creating a new one, with people showing far less reciprocity, show Gachter et al. Accordingly, policies that capitalize on reciprocity in commons maintenance are likely to fail unless economic incentives are offered.

The tragedy of the commons can be averted at multiple scales - but to succeed in this task, we must gain better insight into human behaviour and the reasons behind deciding whether or not to cooperate.

Published online: 12 September 2017 DOI: $10.1038 / \mathrm{s} 41562-017-0210-6$ 